\title{
Measurements in Helical Antennas
}

\author{
Eduardo Rodrigues Vale ${ }^{1}$, Julio Cesar R. Dal Bello ${ }^{2}$ \\ 1,2 (Telecommunications Department, School of Engineering / Federal Fluminense University, Brazil)
}

\begin{abstract}
The circular polarization has some advantages over linear polarization. For example, if it is desirable to obtain a greater immunity to reflections of the radio signal caused by obstacles, one alternative is to use antennas that radiate in circular polarization. For the bands of VHF (Very High Frequency) and UHF (Ultra High Frequency), in this case the most commonly used antenna is the Helical Antenna. To evaluate the electrical characteristics of this type of antennas, we must use a different procedure from that employed for linear polarization antennas. This article will show that, without some additional care, the achievement of parameters such as radiation pattern and gain will present some distortion, thus not depicting the actual electrical behavior of the antenna. In this sense, here it is provided a guide to the effective realization of measures in circular polarization antennas with emphasis on the Helical Antenna.
\end{abstract}

Keywords: Helical Antennas, Circular Polarization, Gain, Radiation Pattern

\section{Introduction}

In Electromagnetics, a known effect is that caused by the incidence of a plane wave of circular polarization into an obstacle. In the case of normal incidence, the reflected wave, also of circular polarization, should possess the reverse direction from that of the incident wave, so as to allow the cancellation of the tangential electric field components on the reflecting surface, thus satisfying the boundary conditions of the electromagnetic phenomenon. If the incidence is not normal, the reflected wave will have elliptical polarization. In any case, since the elliptical polarized wave can be decomposed into two waves with circular polarization in opposite directions [1,7], it can be assumed that a receiving antenna designed to detect the electric field of the incident wave, does not take notice of the wave that rotates in the opposite direction. Thus, it can be assumed that the reflected signal is strongly attenuated at the output of the receiving antenna. This article will evaluate the effects of measures in circularly polarized antennas, performed with techniques commonly employed for measurements of antennas for linear polarization, showing the discrepancies then obtained. In sequence, a roadmap for the correct measurement of gain and radiation pattern of Helical Antennas will be presented.After this introduction, item II presents the expressions of the electric fields radiated by the Helical Antenna; item III gives some consideration aspects of wave polarization; item IV describes the calculation of the theoretical radiation pattern of the Helical Antenna; item V analyzes an application of the theory and item VI presents the conclusions for this article.

\section{Expressions for the Radiation Fields}

The Figure 1 shows the geometry adopted for this section and expressions (1) to (3) present the Radiation Fields (in the Frequency Domain) produced by a Helical Antenna feeded by a current of the form I $\cos \omega t$, where $\omega$ is the angular frequency [1]. Observe that there is no field component in the radial direction, because it is considered that the observation point is very far from the antenna $[2,3,4]$.

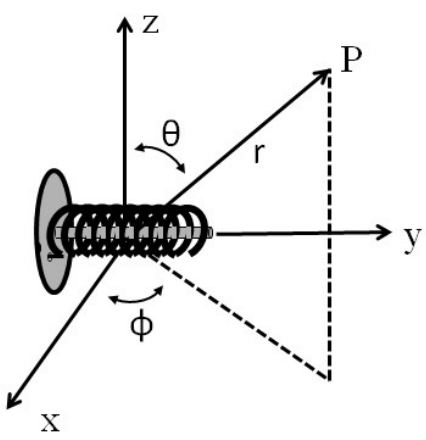

Figure 1: Geometry for the Helical Antenna 


$$
\begin{gathered}
E_{\theta}^{T}=j \frac{30 \pi I e^{-j \beta r}}{r}\left[J_{2}(\sin \phi)-J_{0}(\sin \phi) \frac{\sin (N \Psi / 2)}{\Psi / 2}\right] \\
E_{\phi}^{T}=j \frac{30 \pi I e^{-j \beta r}}{r}\left[J_{2}(\sin \phi)+J_{0}(\sin \phi)\right] \cos \phi \frac{\sin (N \psi / 2)}{\Psi / 2} \\
\Psi=\beta(S \cos \phi-L / P)
\end{gathered}
$$

where:

$\mathrm{N} \rightarrow$ number of turns of the helix;

$\mathrm{P} \rightarrow$ phase velocity of the current wave propagating in the helix $[5,6]$;

$\mathrm{L} \rightarrow$ lenght of one turn of the helix;

$\mathrm{S} \rightarrow$ separation between adjacent turns;

$\mathrm{I} \rightarrow$ current in the antenna input;

$\mathrm{J}_{0}$ and $\mathrm{J}_{2} \rightarrow$ Bessel functions of orders 0 and 2, respectively;

$\beta \rightarrow$ phase constant $(=2 \pi / \lambda)$;

It was considered the expressions for the case of a Helical Antenna with $\mathrm{C}=\lambda(\mathrm{C} \rightarrow$ helix circunference $)$ and $\theta=0^{0}$.

\section{Polarization Considerations}

The fields defined by expressions (1), (2) and (3) are presented, in the most general case, as elliptically polarized plane waves. Just by way of simplification, writing:

$$
\begin{aligned}
& E_{\theta}^{T}=j E_{\theta} e^{-j \beta r} \\
& E_{\phi}^{T}=E_{\phi} e^{-j \beta r}
\end{aligned}
$$

Then, the total field is given by:

$$
E^{T}=\left(j E_{\theta} \bar{u}_{\theta}+E_{\phi} \bar{u}_{\phi}\right) e^{-j \beta r}
$$

As was mentioned, a polarized elliptically field can be decomposed into a sum of two fields circularly polarized in opposite directions. It can be easily checked that the expressions (7) and (8) define fields in these conditions:

$$
\begin{aligned}
& E_{1}=\left(j C \bar{u}_{\theta}+C \bar{u}_{\phi}\right) e^{-j \beta r} \\
& E_{2}=\left(j D \bar{u}_{\theta}-D \bar{u}_{\phi}\right) e^{-j \beta r}
\end{aligned}
$$

The values of $\mathrm{C}$ and $\mathrm{D}$ can be obtained from expressions (6), (7) and (8):

$$
\begin{gathered}
C=\frac{E_{\theta}+E_{\phi}}{2} \\
D=\frac{E_{\theta}-E_{\phi}}{2}
\end{gathered}
$$

Substituting (9) and (10) into (7) and (8) determines the terms of the decomposition of the total field (elliptically polarized) in fields of circular polarization in opposite directions. This development is significant in that it relates the power carried by the elliptically polarized wave with the powers carried by the associated circularly polarized waves.

\section{Radiation Pattern}

The theoretical radiation pattern of the Helical Antenna can be obtained from the general expressions of the fields given by (1), (2) and (3) applied to the Poynting Vector [2], that provides the average radiated power density, ie:

$$
S^{T}=\frac{1}{2} E^{T} \times H^{T^{*}}=\frac{1}{2 \eta}\left(\left|E_{\theta}^{T}\right|^{2}+\left|E_{\phi}^{T}\right|^{2}\right) \bar{u}_{r}
$$

where:

$\mathrm{H} \rightarrow$ Magnetic Field Intensity;

$\eta \rightarrow$ Wave Impedance in Free Space; 
Substituting (1) and (2), it is obtained the expression for the density of power associated with the total (elliptically polarized) radiated wave, ie:

$$
S^{T}=\frac{12|I|^{2}}{r^{2}}\left[\frac{\sin (n \psi / 2)}{\psi / 2}\right]^{2}\left\{\left[J_{2}(\sin \phi)-J_{0}(\sin \phi)\right]^{2}+\left[J_{2}(\sin \phi)+J_{0}(\sin \phi)\right]^{2} \cos ^{2} \phi\right\} \bar{u}_{r}
$$

Substituting (9), (4) e (5) em (7), it determines the expression of the circularly polarized wave component $\left(\mathrm{E}_{1}\right)$ in the right direction. Using (11) we determine the power carried by this right circularly polarized wave:

$$
S_{1}=\frac{6|I|^{2}}{r^{2}}\left[\frac{\sin (n \psi / 2)}{\psi / 2}\right]^{2}\left\{\left[J_{2}(\sin \phi)-J_{0}(\sin \phi)\right]-\left[J_{2}(\sin \phi)+J_{0}(\sin \phi)\right] \cos \phi\right\}^{2} \bar{u}_{r}
$$

\section{Application of the Theory}

As an application of the presented theory, suppose we want to calculate the radiation pattern and the gain of a Helical Antenna having the following parameters:

1. Operation frequency: $300 \mathrm{MHz}$;

2. Helix circunference: $\mathrm{C}=\lambda=100 \mathrm{~cm}$;

3. Angle pitch: $\alpha=12^{0}$;

4. Number of turns of the helix: $\mathrm{N}=8$;

Considering the following definitions [2]:

Substitution of (14), (15) and (16) into (3), gives:

$$
\begin{aligned}
& S=\lambda \tan \alpha \\
& L=\frac{\lambda}{\cos \alpha} \\
& P=\frac{L / \lambda}{\frac{S}{\lambda}+\frac{2 N+1}{2 N}}
\end{aligned}
$$

$$
\psi=1.34 \cos \phi-8.03
$$

Normalizing the expressions (12) and (13) for maximum unit value in the direction of maximum radiation $(\Phi=0)$ we have:

$$
\begin{aligned}
& S N^{T}=\frac{22.43 \sin ^{2}(4 \psi)}{\psi^{2}}\left[\left(J_{2}-J_{0}\right)^{2}+\left(J_{2}+J_{0}\right)^{2} \cos ^{2} \phi\right] \\
& S N^{1}=\frac{11.21 \sin ^{2}(4 \psi)}{\psi^{2}}\left[\left(J_{2}-J_{0}\right)-\left(J_{2}+J_{0}\right) \cos \phi\right]^{2}
\end{aligned}
$$

where it was used the definitions:

$\mathrm{J}_{0} \rightarrow \mathrm{J}_{0}(\sin \Phi)$

$\mathrm{J}_{2} \rightarrow \mathrm{J}_{2}(\sin \Phi)$

Finally, by making:

$$
\begin{aligned}
& S N_{d B}^{T}=10 \log S N^{T} \\
& S N_{d B}^{1}=10 \log S N^{1}
\end{aligned}
$$

The expressions (20) and (21) provide the radiation patterns of a Helical Antenna for the total radiated power and the amount of power carried only by the right circularly polarized wave.

Figure 2 illustrates these diagrams, with the solid line being the actual antenna radiation pattern and the dotted line the pattern seen by a Helical Antenna, operating as a receiver, with his helix winding constructed to the right. As might be expected, for $\Phi>80^{\circ}$ we note a big difference between the patterns. As commented before, this effect will be reflected directly on the antenna gain, if it is calculated by the method of pattern integration. 


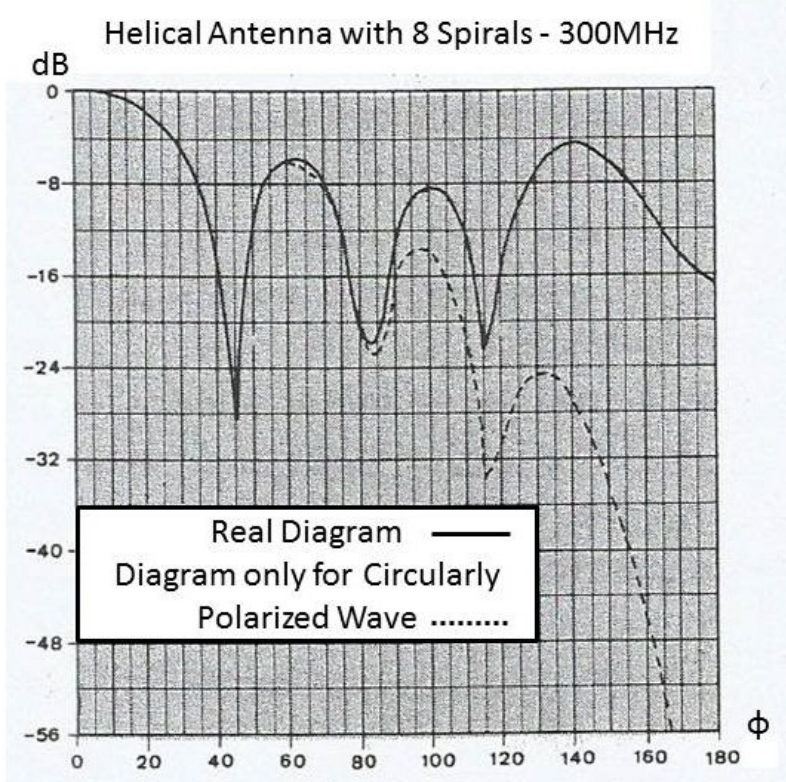

Figure 2: Radiation Patterns of a Helical Antenna

In order to compare the results, the gains were calculated for the two diagrams, taking points with angular offset of $1^{0}$ (360 points), from the use of the following expressions [1]:

$$
\begin{gathered}
G_{d B}^{T}=10 \log \frac{720}{\pi \sum_{\phi=1}^{360} S N^{T} \sin \phi} \\
G_{d B}^{1}=10 \log \frac{720}{\pi \sum_{\phi=1}^{360} S N^{1} \sin \phi}
\end{gathered}
$$

Calculating the expressions (22) and (23) by computer, it was obtained:

$$
\begin{aligned}
G_{d B}^{T} & =7.8 d B \\
G_{d B}^{1} & =10.0 d B
\end{aligned}
$$

This result demonstrates, again, the error that may be committed for determining the gain of circular polarization antennas through the method of pattern integration.

\section{Conclusions}

According to the results, it is observed that a radiation pattern of a Helical Antenna (or any other with circular polarization), when obtained from two similar antennas, in a testing field, only provides reliable values for angles in the range $-90^{\circ}$ to $+90^{\circ}$.

The determination of the Helical Antenna gain parameter, with the integration pattern method, leads to totally unrealistic values, always one obtaining better values than those observed with the absolute measurement process [1].

Therefore, a simple and accurate process for determining the radiation patterns of antennas which operate with circular polarization, is defined by the following steps:

1. Determine the radiation pattern, on a test field, with two identical antennas, one at the transmission side and the other at the reception;

2. Reverses the direction of polarization of one antenna and draw a new radiation pattern;

3. Add the outcomes and normalize the result;

\section{References}

[1]. E. R. Vale and J. C. R. Dal Bello, Correction of Measurements in Circular Polarized Antennas, IOSR Journal of Electronics and Communications Engineering,vol. 9, issue 4, july 2014.

[2]. Kraus and Carver, Electromagnetics (New York: Mc Graw Hill, 1965).

[3]. J. D. Kraus, Characteristics of Helical Antennas Radiating in the Axial Mode, Journal of Applied Physics, v19, jan 1947, 87-96. 
[4]. J. D. Kraus, The Helical Antenna, Proceedings of the IRE, March 1949, 263-272.

[5]. I. Inagaki and Sekiguchi, An Investigation of the Array of Circular-Loop Antennas, IEEE Transactions on Antennas and Propagation, AP-19, nº July 1971 .

[6]. Lindsay and J. E. Junior, A Circular Loop Antenna with Nonuniform Current Distribution, IRE Transactions on Antennas and Propagation, July 1960, 439-441.

[7]. E. R. Vale, Characteristics of Circular Polarized Antennas, Revista Telebrás, dez 1980, 50-56. 